\begin{tabular}{|c|c|}
\hline hommes & $\begin{array}{l}\text { Hommes \& migrations } \\
\text { Revue française de référence sur les dynamiques } \\
\text { miqratoires }\end{array}$ \\
\hline & $\begin{array}{l}1281 \text { | } 2009 \\
\text { France-Brésil sous l'angle des migrations et de } \\
\text { l'altérité }\end{array}$ \\
\hline
\end{tabular}

\title{
Dans le sillon de Claude Lévi-Strauss
}

\section{Marie Poinsot}

\section{(2) OpenEdition}

Journals

Édition électronique

URL : http://journals.openedition.org/hommesmigrations/364

DOI : 10.4000/hommesmigrations.364

ISSN : 2262-3353

Éditeur

Musée national de l'histoire de l'immigration

Édition imprimée

Date de publication : 1 septembre 2009

Pagination : 1

ISSN : 1142-852X

\section{Référence électronique}

Marie Poinsot, «Dans le sillon de Claude Lévi-Strauss », Hommes \& migrations [En ligne], 1281 | 2009,

mis en ligne le 29 mai 2013, consulté le 22 septembre 2020. URL : http://journals.openedition.org/

hommesmigrations/364 ; DOI : https://doi.org/10.4000/hommesmigrations.364 


\section{Dans le sillon de Claude Lévi-Strauss \\ Par Marie Poinsot, rédactrice en chef}

L'Année de la France au Brésil a suscité un vif intérêt de part et d'autre de l'Atlantique ; pour preuve l'accueil réservé à l'exposition "A Imigraçao na França : pontos de referência" réalisée par la Cité nationale de l'histoire de l'immigration au Mémorial des migrants à São Paulo cet automne.

Pour l'occasion, la revue a lancé un appel à contributions portant sur les études comparées entre ces deux pays sur les migrations et les relations interculturelles dont les contextes historiques apparaissent pourtant très différents. La coopération avec des organismes comme le Centre de recherche sur le Brésil de l'EHESS, le Musée national de l'université fédérale de Rio de Janeiro, l'université du Ceará - Fortaleza, le Mémorial des migrants et le CEMPS a permis de rassembler de nombreux articles sous la coordination d'Abdelhafid Hammouche dont on remercie l'investissement particulier. La rédaction a proposé à la revue brésilienne Travessia, la revue du migrant, éditée par le Centre des études migratoires à São Paulo, de publier un hors série à partir des articles qu'Hommes et Migrations avait retenu en complément du dossier.

Ce partenariat éditorial souligne les affinités intellectuelles anciennes qui unissent le Brésil et la France dans le domaine des sciences sociales. Si le Brésil fut le premier terrain d'études de Claude Lévi-Strauss, aujourd'hui disparu, et dont la revue rend ici le plus chaleureux hommage, il fut aussi la terre de prédilection de l'ethnologie française naissante à travers les travaux de Roger Bastide, de Pierre Berger et d'autres spécialistes des relations raciales, des transferts culturels et des religions dans cette société multiple, forte d'une histoire complexe issue des vagues migratoires successives. Leur expérience intime avec la société brésilienne fut favorisée par la proximité rendue possible avec les peuples étudiés mais aussi par un accueil enthousiaste dans les milieux académiques brésiliens, francophones, francophiles, attentifs aux approches théoriques que ces intellectuels français allaient continuer à développer de retour en France à partir de leurs années brésiliennes. Leurs analyses des cultures du Brésil constituent de ce fait des repères pour la compréhension des relations interculturelles dans la société française. Cet héritage commun entre le Brésil et la France, fortifié de nouveau dans les années quatre-vingt-dix par les deux séjours d'Abdelmalek Sayad au Musée national de Rio de Janiero, reste extrêmement vivant de nos jours, grâce aux échanges permanents entre les chercheurs de ces deux pays. Les questionnements des uns renouvellent les pratiques sociologiques des autres tout en dessinant de nouveaux objets de recherche sur les migrations. Ce dossier met en lumière les avantages de la circulation des problématiques et des méthodes d'enquêtes qui se concrétisent à travers les déplacements des chercheurs et la diffusion de leurs travaux. Dans le sillon de Claude Lévi-Strauss, qui initia cette relation privilégiée entre le Brésil et la France, on mesure mieux l'importance de soumettre chaque modèle explicatif au regard extérieur des chercheurs travaillant sur une société différente. Cette nécessaire distance, comme mise en perspective, dessine un "internationalisme scientifique" auquel les revues Hommes et Migrations, Travessia et tant d'autres tentent à leur manière de contribuer. 